\title{
GEOMETRY OF THE RECURSION OPERATORS FOR CAUDREY- BEALS-COIFMAN SYSTEM IN THE PRESENCE OF MIKHAILOV TYPE $\mathbb{Z}_{P}$ REDUCTIONS
}

\author{
ALEXANDAR B. YANOVSKI
}

Presented by Alexandar B. Yanovski

Abstract. We give geometric picture for the Recursion Operators related to the Caudrey-Beals-Coifman linear problem and the Nonlinear Evolution Equations associated to it in the presence of $\mathbb{Z}_{p}$ reductions of Mikhailov type.

\section{Introduction}

It is well known that the characteristic property of the nonlinear evolution equations (NLEEs) of soliton type is that they admit the so called Lax representation $[L, A]=0$. In the last expression $L, A$ are linear operators on $\partial_{x}, \partial_{t}$ depending also on a family of functions $q_{\alpha}(x, t), 1 \leq \alpha \leq s$ (called 'potentials') and a spectral parameter $\lambda$. Since the Lax equation $[L, A]=0$ must be satisfied identically in $\lambda$ it is equivalent to a system (in case $A$ depends linearly on $\partial_{t}$ ) of the type

$$
\left(q_{\alpha}\right)_{t}=F_{\alpha}\left(q, q_{x}, \ldots\right), \quad \text { where } \quad q=\left(q_{\alpha}\right)_{1 \leq \alpha \leq s}
$$

which is the NLEE (soliton equation) associated with $A$ and $L$. Usually one fixes the linear problem $L \psi=0$ (auxiliary linear problem) and considers all the NLEEs (of certain form) that can be obtained changing the operator $A$. There are various schemes used to find exact solutions to a given soliton equation but the essential is that the Lax representation permits to pass from the original evolution defined by the equations (1) to the evolution of some spectral data related to the problem $L \psi=0$. Since finding the evolution on the spectral data is easy, the principal difficulty is then to recover from the spectral data the potentials, a process called Inverse Scattering Method, see the monograph books [4, 8].

The Caudrey-Beals-Coifman system (CBC system), [2], called also the Generalized Zakharov-Shabat system in the case when the element $J$ is real, is one of the best known auxiliary linear problems. It can be written as follows

$$
L \psi=\left(\mathrm{i} \partial_{x}+q(x)-\lambda J\right) \psi=0 .
$$


Originally it has been assumed that $J$ is a fixed complex $n \times n$ diagonal matrix and $q(x)$ is a matrix function taking values in the space of the off-diagonal matrices. Soon however it has been understood that the theory can be developed for the case when $q(x)$ and $J$ belong to a fixed simple Lie algebra $\mathfrak{g}$ in some finite dimensional irreducible representation. The element $J$ should be regular, that is the kernel of $\operatorname{ad}_{J}\left(\operatorname{ad}_{J}(X) \equiv[J, X], X \in \mathfrak{g}\right)$ is the Cartan subalgebra $\mathfrak{h} \subset \mathfrak{g}$. The potential $q(x)$ belongs to the orthogonal complement $\mathfrak{h}^{\perp}=\overline{\mathfrak{g}}$ of $\mathfrak{h}$ with respect to the Killing form

$$
\langle X, Y\rangle=\operatorname{tr}\left(\operatorname{ad}_{X} \operatorname{ad}_{Y}\right), \quad X, Y \in \mathfrak{g}
$$

and therefore $q(x)=\sum_{\alpha \in \Delta} q_{\alpha} E_{\alpha}$ where $E_{\alpha}$ are the root vectors and $\Delta$ is the root system of $\mathfrak{g}$. The scalar functions $q_{\alpha}(x)$ (the 'potentials') are defined on $\mathbb{R}$, are complex valued, smooth and tend to zero as $x \rightarrow \pm \infty$. We shall suppose that they are Schwartz-type functions. The classical Zakharov-Shabat system is obtained for $\mathfrak{g}=\mathfrak{s l}(2, \mathbb{C}), J=\operatorname{diag}(1,-1)$.

Remark 1. We assume that the basic properties of the semisimple Lie algebras are known and we do not give definitions to all the notions related to them. All our definitions and normalizations coincide with those made in [13].

The spectral theory of $L$ is of a primary importance for the development of the inverse scattering techniques for $L$ and has been object of many studies. We mention [7], in which it is proved the completeness of the so-called adjoint solutions of $L$ when $L$ is considered in arbitrary faithful representation of the algebra $\mathfrak{g}$. Without going into details we remind briefly the main facts. First, the adjoint solutions of $L$ are functions of the type $w=m X m^{-1}$ where $X$ is a constant element from $\mathfrak{g}$ and $m$ is fundamental solution of $L m=0$. Suppose we denote by $w^{\mathrm{a}}$ and $w^{\mathrm{d}}$ the orthogonal projection (with respect to the Killing form) of $w$ over $\mathfrak{h}^{\perp}$ and $\mathfrak{h}$ respectively. If one denotes the orthogonal projector on $\mathfrak{h}^{\perp}$ by $\pi_{0}$ then of course $w^{\mathrm{a}}=\pi_{0} w$ and $w^{\mathrm{d}}=\left(\mathrm{id}-\pi_{0}\right) w$. One of the most important facts from the theory of CBC system is that if a suitable set of adjoint solutions $\left(w_{i}(x, \lambda)\right)$ is taken then, roughly speaking, for $\lambda$ on the spectrum of $L$ the functions $w_{i}^{\mathrm{a}}(x, \lambda)$ form a complete set in the space of potentials. If one expands the potential over the subset of the adjoint solutions as coefficients one gets the minimal scattering data for $L$. Thus passing from the potentials to the scattering data can be considered as a sort of Fourier Transform, called Generalized Fourier Transform. For it $w_{i}^{\mathrm{a}}(x, \lambda)$ play the role the exponents play in the Fourier Transform. This interpretation of the Inverse Scattering Transform has been given for the first time in [1] and after that has been developed in a number of works, see for example the monograph books $[8,16]$ for complete study of $\mathfrak{s l}(2, \mathbb{C})$-case and comprehensive bibliography, and $[2,7]$ for more general situations. 
One of the ways the Recursion Operators (Generating Operators, $\Lambda$-operators) can be found is motivated by the desire to find operators for which the functions $w_{i}^{\mathrm{a}}(x, \lambda)$ are eigenfunctions. One can see that for the Generalized Fourier Transform such operators play the same role as the differentiation operator in the usual Fourier Transform method. Because of that property the Recursion Operators are so important in the theory of soliton equations - it is a theoretical too which apart from explicit solutions can give most of the information about the NLEEs, $[8,30]$. Indeed, through them can be obtained

i) The hierarchies of the nonlinear evolution equations solvable through $L$

ii) The conservation laws for these NLEEs

iii) The hierarchies of Hamiltonian structures for these NLEEs.

It is not hard to get that the Recursion Operators related to $L$ have the form

$$
\begin{aligned}
& \Lambda_{ \pm}(X(x))= \\
& \operatorname{ad}_{J}^{-1}\left(\mathrm{i} \partial_{x} X+\pi_{0}[q, X]+\operatorname{iad}_{q} \int_{ \pm \infty}^{x}\left(\mathrm{id}-\pi_{0}\right)[q(y), X(y)] \mathrm{d} y\right)
\end{aligned}
$$

where of course $\operatorname{ad}_{q}(X)=[q, X]$ and $X$ is a smooth, fast decreasing function with values in $\mathfrak{h}^{\perp}=\overline{\mathfrak{g}}$. We call the above operators the Recursion Operators for the CBC system in general position.

The name Recursion Operators has the following origin and gives an alternative definition for them. Suppose we are looking for the NLEEs that have Lax representation $[L, A]=0$ with $L$ given in (2) and $A$ of the form

$$
\begin{aligned}
& A=\mathrm{i} \partial_{t}+\sum_{k=0}^{n} \lambda^{k} A_{k} \\
& A_{n} \in \mathfrak{h}, \quad A_{n}=\text { const }, \quad A_{n-1} \in \mathfrak{h}^{\perp}=\overline{\mathfrak{g}} .
\end{aligned}
$$

Then from the condition $[L, A]=0$ we first obtain $A_{n-1}=\operatorname{ad}_{J}^{-1}[q, A]$ and for $0<k<n-1$ the recursion relations

$$
\begin{aligned}
& \pi_{0} A_{k-1}=\Lambda_{ \pm}\left(\pi_{0} A_{k}\right) \\
& \left(\mathrm{id}-\pi_{0}\right) A_{k}=\mathrm{i}\left(\mathrm{id}-\pi_{0}\right) \int_{ \pm \infty}^{x}\left[q, \pi_{0} A_{k}\right](y) \mathrm{d} y
\end{aligned}
$$


This leads to the fact that the NLEEs related to $L$ can be written into one of the following equivalent forms

$$
\begin{aligned}
& \text { a) } \operatorname{iad}_{J}^{-1} q_{t}+\Lambda_{+}^{n}\left(\operatorname{ad}_{J}^{-1}\left[A_{n}, q\right]\right)=0 \\
& \text { b) } \operatorname{iad}_{J}^{-1} q_{t}+\Lambda_{-}^{n}\left(\operatorname{ad}_{J}^{-1}\left[A_{n}, q\right]\right)=0 .
\end{aligned}
$$

Remark 2. Strictly speaking the above is not the general form of the equations solvable through L. Considering the right-hand side of the equations of the type $\operatorname{ad}_{\mathrm{J}}^{-1} \mathrm{q}_{\mathrm{t}}=\mathrm{F}_{\mathrm{n}}(\mathrm{q})$ as vector fields in order to obtain the general form of the NLEEs associated with $L$ one must take arbitrary finite linear combination $F$ of the vector fields $F_{n}$ with constant coefficients and write $\mathrm{ad}_{\mathrm{J}}^{-1} \mathrm{q}_{\mathrm{t}}=\mathrm{F}(\mathrm{q})$. We refer to (8) as the general form of the equations solvable through $L$ for the sake of brevity.

The Recursion Operators have also interesting geometric interpretation (their adjoint operators can be interpreted as Nijenhuis tensors on the manifold of potentials). The present article is dedicated to this subject, more precisely, what happens with the geometry of the Recursion Operators in the presence of Mikhailov-type reductions. The implications of the Mikhailov-type reductions on the theory of Recursion Operators been considered recently in several papers, [10-12,27,29] but they rather treat the case of the $\mathrm{CBC}$ system in pole gauge and from the viewpoint of the recursion relations and spectral theory. The CBC system in canonical gauge (the one we shall discuss) subject to reductions has been considered much earlier. For example, in $[14,15]$ were investigated the implications to the scattering data. In [9] the Recursion Operators has been considered from spectral theory viewpoint. In the present article we give a treatment from geometric viewpoint, similar to that given [28] for the CBC in pole gauge in general position.

At the beginning let us briefly outline the reduction procedures for the $\mathrm{CBC}$ system and the NLEEs related to it.

\section{The Mikhailov-Type $\mathbb{Z}_{p}$ Reductions}

\subsection{General Remarks}

Consider the linear problems of the type (2) in which the potential function $q(x)$ and the element $J$ obey some special requirements. These requirements result from Mikhailov-type reductions, see [22-24]. The reduction problem naturally appears for example if we want to consider real potentials instead of complex ones or if we want drastically to decrease the number of the scalar equations in the integrable equation (system) that is related to a CBC system which we consider 
defined on a simple complex Lie algebra $\mathfrak{g}$. We shall consider reductions related with automorphisms of $\mathfrak{g}$ of order $p-\mathbb{Z}_{p}$ reductions. We shall assume that the automorphisms leave invariant the Cartan subalgebra $\mathfrak{h} \subset \mathfrak{g}$ to which the element $J$ in the CBC system belongs. As natural examples for automorphisms of fixed order we consider the Coxeter automorphisms.

Suppose $\mathcal{K}$ is an automorphism of $\mathfrak{g}$ of order $p$, that is $\mathcal{K}^{p}=\mathrm{id}, \mathcal{K} \mathfrak{h} \subset \mathfrak{h}$. (In case $\mathcal{K}$ is Coxeter automorphisms $p$ is called the Coxeter number). The Coxeter automorphisms are internal, that is, each $\mathcal{K}$ can be represented as $\mathcal{K}=\operatorname{Ad}(K)$, $K$ belonging to the corresponding group $G$ with algebra $\mathfrak{g}$. Thus $\mathcal{K}$ can be integrated' to act on $G$ by $\mathcal{K}(g)=K g K^{-1}$. Arbitrary automorphism needs not to be internal but to each automorphism $\mathcal{K}$ of finite order corresponds automorphism of the corresponding Lie group $G$ which we shall denote by the same letter. Also, we remind also that automorphisms leave the Killing form invariant, a fact that we shall use constantly.

The algebra $\mathfrak{g}$ splits into a direct sum of eigenspaces of $\mathcal{K}$, that is

$$
\mathfrak{g}=\oplus_{s=0}^{p-1} \mathfrak{g}^{[s]}
$$

where for each $X \in \mathfrak{g}^{[s]}$ we have $\mathcal{K} X=\omega^{s} X, \omega=\exp \frac{2 \pi \mathrm{i}}{p}$ and the spaces $\mathfrak{g}^{[s]}, \mathfrak{g}^{[k]}$ for $k \neq s$ are orthogonal with respect to the Killing form. Because $\mathcal{K}$ is an automorphism of $\mathfrak{g}$ leaving $\mathfrak{h}$ invariant, it leaves invariant also the orthogonal complement $\overline{\mathfrak{g}}$ of $\mathfrak{h}$. Thus each $\mathfrak{g}^{[s]}$ splits into $\overline{\mathfrak{g}}^{[s]} \oplus \mathfrak{h}^{[s]}$ and

$$
\overline{\mathfrak{g}}=\oplus_{s=0}^{p-1} \overline{\mathfrak{g}}^{[s]}, \quad \mathfrak{h}=\oplus_{s=0}^{p-1} \mathfrak{h}^{[s]}
$$

For different $k$ and $s$ the spaces $\mathfrak{g}^{[k]}$ and $\mathfrak{g}^{[s]}$ are orthogonal with respect to the Killing form and the spaces $\overline{\mathfrak{g}}^{[k]}$ and $\mathfrak{h}^{[s]}$ are orthogonal for arbitrary $k$ and $s$. Further, if we denote the orthogonal projections onto $\mathfrak{g}^{[k]}$ by $\mathbf{1}^{[k]}$ we shall have that $\zeta^{[k]}=\mathbf{1}^{[k]}\left(\mathbf{1}-\pi_{0}\right)$ are the projections onto $\mathfrak{h}^{[k]}$ and $\mathbf{1}^{[k]} \pi_{0}=\pi_{0}^{[k]}$ are the orthogonal projector onto $\overline{\mathfrak{g}}^{[k]}$.

If as before the orthogonal projector $\mathfrak{g} \mapsto \overline{\mathfrak{g}}$ is denoted by $\pi_{0}$ we shall have

$$
\begin{array}{rlrl}
\pi_{0} & =\sum_{k=0}^{p-1} \pi_{0}^{[k]}, & & \pi_{0}^{[l]} \pi_{0}^{[s]}-\pi_{0}^{[s]} \pi_{0}^{[l]}=0 \\
\mathbf{1}-\pi_{0} & =\sum_{k=0}^{p-1} \zeta^{[k]}, & \zeta^{[l]} \zeta^{[s]}-\zeta^{[s]} \zeta^{[l]}=0 \\
\pi_{0}^{[k]}+\zeta^{[k]} & =\mathbf{1}^{[k]}, & \zeta^{[l]} \pi_{0}^{[s]}=\pi_{0}^{[s]} \zeta^{[l]}=0 .
\end{array}
$$


Let us consider Mikhailov reduction group $G_{0}$ is generated by one element, which we denote by $H$. On the fundamental solutions $\psi$ of the CBC system it acts as

$$
H(\psi(x, \lambda))=\mathcal{K}\left(\psi\left(x, \omega^{-1} \lambda\right)\right)
$$

where $\omega=\exp \frac{2 \pi \mathrm{i}}{p}$. Since $H^{p}=\mathrm{id}$ the reduction group $G_{0}$ is isomorphic to $\mathbb{Z}_{p}$. Let us assume that the set of fundamental solutions for the spectral problem (2) is invariant under $G_{0}$, that is, $H(\psi(x, \lambda))$ is a fundamental solution corresponding to the same value of $\lambda$. Then as it is easy to see that we must have

$$
\mathcal{K}(J)=\omega J, \quad \mathcal{K} q=q
$$

that is, $J \in \mathfrak{g}^{[1]}, q(x) \in \mathfrak{g}^{[0]}$. In fact, suppose we have a Lax representation $[L, A]=0$ where $A$ has the form

$$
\begin{aligned}
& A=\mathrm{i} \partial_{t}+\sum_{k=0}^{n} \lambda^{k} A_{k} \\
& A_{n} \in \mathfrak{h}, \quad A_{n}=\text { const }, \quad A_{n-1} \in \overline{\mathfrak{g}} .
\end{aligned}
$$

If the set of the mutual fundamental solutions for $L \psi=0, A \psi=0$ is invariant under $G_{0}$ then we also have

$$
\mathcal{K}\left(A_{s}\right)=\omega^{s} A_{s}, \quad s=0,1,2, \ldots n .
$$

The above reductions are compatible with the evolution in the sense that if at the moment $t=0$ we have $(15,17)$ we have the same relations at arbitrary moment $t$. The invariance of the set of the fundamental solutions has important implications on the spectral theory of the CBC system and of the Recursion Operators but in this article we are not going to address this matter. We shall only mention that in case of reductions there are relations between the fundamental analytic solutions $m_{\nu}(x, \lambda)$ defined on the closures of the sectors of analyticity $\Omega_{\nu}, \nu=1,2, \ldots h$. The sectors $\Omega_{\nu}$ are obtained splitting the complex plane by the straight lines

$$
l_{\alpha}=\{\lambda ; \operatorname{Im}(\lambda \alpha(J))=0\}, \quad \alpha \in \Delta
$$

where Im denotes imaginary part. Of course, one obtains the same line for $\alpha$ and $-\alpha$ but it can happen that $\alpha \neq \beta$ and $l_{\alpha}=l_{\beta}$. Also, the number of the sectors depends on the order of the automorphism.

Let us recall that since $\mathcal{K}$ preserves the Cartan subalgebra $\mathfrak{h}$ its dual map $\mathcal{K}^{*}$ preserves $\mathfrak{h}^{*}$. For $H \in \mathfrak{h}$ we define $\hat{\mathcal{K}}: \mathfrak{h} \mapsto \mathfrak{h}$ by $\hat{\mathcal{K}}=\left(\mathcal{K}^{*}\right)^{-1}$. For $\mathcal{K}$ that is internal (of the form $X \mapsto K X K^{-1}$ ) one of course has $\hat{\mathcal{K}}(\alpha)(H)=\alpha\left(K^{-1} H K\right)$. 
The map $\hat{\mathcal{K}}$ defines the coadjoint action of $\mathcal{K}$ on $\mathfrak{h}^{*}$. Naturally $\hat{\mathcal{K}}^{p}=$ id and for $\xi \in \mathfrak{h}^{*}, H \in \mathfrak{h}$ one has

$$
\langle\hat{\mathcal{K}} \xi, \mathcal{K} H\rangle=\langle\xi, H\rangle .
$$

It can be shown, see [13], that for all roots we have $\mathcal{K} E_{\alpha}=q(\alpha) E_{\hat{\mathcal{K}} \alpha}$, where $q(\alpha)= \pm 1$ and $q(\alpha) q(-\alpha)=1$ and from the map $\hat{\mathcal{K}}$ one can completely recover the automorphism $\mathcal{K}$.

\subsubsection{Coxeter Automorphisms}

As an example consider reductions that arise using Coxeter automorphisms. In fact when $\mathcal{K}$ is a Coxeter automorphism the situation is quite simple because the requirement for $J$ determines it up to a constant nonzero multiplier, that is $\mathfrak{g}^{[1]} \cap$ $\mathfrak{h}$ is one-dimensional. In order to see it we shall make some preparations. We remind that the Coxeter automorphisms are the automorphisms for which $\hat{\mathcal{K}}=$ $S_{\alpha_{1}} S_{\alpha_{2}} \ldots S_{\alpha_{r}}$ where $S_{\alpha_{i}}$ are the Weyl reflections corresponding to the simple roots $\alpha_{1}, \alpha_{2}, \ldots, \alpha_{r}$ of $\mathfrak{g}$ (we assume that the Cartan subalgebra $\mathfrak{h}$ is fixed and a system of simple roots is fixed too).

If we need only the action of $\hat{\mathcal{K}}$ on the root system it is easier to work in $\mathfrak{h}^{*}$ than in $\mathfrak{h}$. Then it is natural to consider instead of $J$ the element $J^{*}$ in $\mathfrak{h}^{*}$ such that $J^{*}(H)=$ $\langle J, H\rangle, H \in \mathfrak{h}$. As the Cartan-Killing form is invariant under the automorphisms, $\hat{\mathcal{K}}\left(J^{*}\right)(H)=\langle J, \mathcal{K}(H)\rangle$ and the condition $\mathcal{K}(J)=\omega J$ is equivalent to $\hat{\mathcal{K}}\left(J^{*}\right)=$ $\bar{\omega} J^{*}$. Now, from the definition of the coadjoint action of the automorphism $\mathcal{K}$, taking into account that $J=\sum_{i=1}^{r} a_{i} H_{\alpha_{i}}$, where $\left\langle H_{\alpha_{i}}, H\right\rangle=\alpha_{i}(H)$ for $H \in \mathfrak{h}$, we obtain that for $\beta \in \Delta$

$$
\beta(J)=\sum_{i=1}^{r} a_{i} \beta\left(H_{\alpha_{i}}\right)=\sum_{i=1}^{r} a_{i}\left(\beta, \alpha_{i}\right)=\left(J^{*}, \beta\right)
$$

where $J^{*}=\sum_{i=1}^{r} a_{i} \alpha_{i}$. Then $\alpha\left(K J K^{-1}\right)=(\hat{\mathcal{K}} \alpha)(J)=\left(\hat{\mathcal{K}} \alpha, J^{*}\right)$. If in addition $K J K^{-1}=\omega J$ then $K^{-1} J K=\bar{\omega} J$, where $\bar{\omega}=\omega^{-1}=\exp \frac{-2 \pi \mathrm{i}}{p}$. As a consequence

$$
\left(\hat{\mathcal{K}} \alpha, J^{*}\right)=\bar{\omega} \alpha(J)=\bar{\omega}\left(\alpha, J^{*}\right), \quad \alpha \in \Delta .
$$

$\operatorname{But}\left(\hat{\mathcal{K}} \alpha, J^{*}\right)=\left(\alpha, \hat{\mathcal{K}} J^{*}\right)$ and we deduce that the requirement $K J K^{-1}=\omega J$ is equivalent to

$$
\hat{\mathcal{K}} J^{*}=\bar{\omega} J^{*} .
$$

This equation is more convenient in order to calculate the coefficients $a_{i}$ and going on all the simple Lie algebras one can check that $J$ is defined up to a nonzero 
multiplier. As an example, consider the series $C_{n} \sim \mathfrak{s p}(n, \mathbb{C})$. The root space is realized as subset of an Euclidean space spanned by vectors $\epsilon_{i}, 1 \leq i \leq n$ such that:

$$
\left(\epsilon_{i}, \epsilon_{j}\right)=\frac{\delta_{i j}}{4(n+1)} .
$$

The simple roots are $\alpha_{i}=\epsilon_{i}-\epsilon_{i+1}, 1 \leq i \leq n-1, \alpha_{n}=2 \epsilon_{n}$ and the set of roots $\Delta$ consists of $\epsilon_{i}-\epsilon_{j}, i \neq j, \epsilon_{i}+\epsilon_{j}, 1 \leq i, j \leq n$. We easily check that

$$
\hat{\mathcal{K}} \epsilon_{1}=\epsilon_{2}, \quad \hat{\mathcal{K}} \epsilon_{2}=\epsilon_{3}, \quad \ldots, \quad \hat{\mathcal{K}} \epsilon_{n-1}=\epsilon_{n}, \quad \hat{\mathcal{K}} \epsilon_{n}=-\epsilon_{1}
$$

and as a consequence

$$
\hat{\mathcal{K}} \alpha_{1}=\alpha_{2}, \quad \hat{\mathcal{K}} \alpha_{2}=\alpha_{3}, \quad \ldots, \quad \hat{\mathcal{K}} \alpha_{n-1}=\sum_{i=1}^{n} \alpha_{i}, \quad \hat{\mathcal{K}} \sum_{i=1}^{n} \alpha_{i}=-\alpha_{1} .
$$

So the Coxeter number is $p=2 n$ and $\omega=\exp \frac{2 \pi \mathrm{i}}{2 n}=\exp \frac{\pi \mathrm{i}}{n}$. We easily get that

$$
J^{*}=a \sum_{i=1}^{n}\left(\omega^{i-1} \epsilon_{i}\right), \quad a=\text { const. }
$$

\section{Recursion Operators and $\mathbb{Z}_{p}$ Reductions}

Let us see now what happens with the Recursion Operator when $\mathbb{Z}_{p}$ reductions are present. As we have seen the Recursion Operator for the system in general position (without reductions) can be written as

$$
\left.\Lambda_{ \pm} X=\operatorname{ad}_{J}^{-1}\left\{\mathrm{i} \partial_{x} X+\pi_{0}[q, X]+\operatorname{iad}_{q}\left(\mathbf{1}-\pi_{0}\right) \partial_{x}^{-1}[q, X]\right\rangle\right\} .
$$

Now assume that we have $\mathbb{Z}_{p}$ reduction. Then the algebra splits in a direct sum, see (9), and $q \in \mathfrak{g}^{[0]}$ while $J \in \mathfrak{h}^{[1]}$. In particular, this means that

$$
\operatorname{ad}_{J}\left(\overline{\mathfrak{g}}^{[s]}\right) \subset \overline{\mathfrak{g}}^{[s+1]}, \quad \operatorname{ad}_{J}^{-1}\left(\overline{\mathfrak{g}}^{[s]}\right) \subset \overline{\mathfrak{g}}^{[s-1]} .
$$

Here and below we shall understand the superscripts of the spaces and the subscripts of the operators modulo $p$. Also, if $X \in \overline{\mathfrak{g}}^{[s]}$ then $\partial_{x} X \in \overline{\mathfrak{g}}^{[s]}, \partial_{x}^{-1} X \in \overline{\mathfrak{g}}^{[s]}$, $[q, X] \in \overline{\mathfrak{g}}^{[s]}$ and

$$
\Lambda_{ \pm} X=\operatorname{ad}_{J}^{-1}\left\{\mathrm{i} \partial_{x} X+\pi_{0}[q, X]+\operatorname{ad}_{q} \partial_{x}^{-1}\left(\mathbf{1}-\pi_{0}\right)[q, X]\right\} \in \overline{\mathfrak{g}}^{[s-1]} .
$$

If we use the notation introduced in (11) the above expression can also be written as

$$
\Lambda_{ \pm} X=\operatorname{ad}_{J}^{-1}\left\{\operatorname{i}_{x}+\pi_{0} \operatorname{ad}_{q}+\operatorname{ad}_{q} \partial_{x}^{-1}\left(\mathbf{1}-\pi_{0}\right) \operatorname{ad}_{q}\right\} \pi_{0}^{[s]} X .
$$


Let us denote the right-hand side of this equality by $\Lambda_{ \pm ; s} X$. As easily seen it defines an operator $\Lambda_{ \pm ; s}$ acting on the space $\mathfrak{F}(\overline{\mathfrak{g}})$ consisting of smooth, rapidly decreasing functions with values in $\overline{\mathfrak{g}}$. The spaces $\mathfrak{F}\left(\overline{\mathfrak{g}}^{[s]}\right)$ consisting of rapidly decreasing smooth functions $X(x)$ with values in $\overline{\mathfrak{g}}^{[s]}$ are moved one into another by $\Lambda_{ \pm}$and are invariant under the action of $\Lambda_{ \pm}^{p}$. Naturally

$$
\left.\Lambda_{ \pm}\right|_{\mathfrak{F}\left(\overline{\mathfrak{g}}^{[s]}\right)}=\left.\Lambda_{ \pm ; s}\right|_{\mathfrak{F}\left(\overline{\mathfrak{g}}^{[s]}\right)}, \quad \Lambda_{ \pm ; s} \mathfrak{F}\left(\overline{\mathfrak{g}}^{[s]}\right) \subset \mathfrak{F}\left(\overline{\mathfrak{g}}^{[s-1]}\right)
$$

Also

$$
\left.\Lambda_{ \pm}^{p}\right|_{\mathfrak{F}\left(\overline{\mathfrak{g}}^{[s]}\right)}=\Lambda_{ \pm ; p-s+1} \ldots \Lambda_{ \pm ; s-1} \Lambda_{ \pm ; s}
$$

in which the indices are understood modulo $p$ here. In particular

$$
\left.\Lambda_{ \pm}^{p}\right|_{\mathfrak{F}(\overline{\mathfrak{g}}[0])}=\Lambda_{ \pm ; 1} \ldots \Lambda_{ \pm ; p-2} \Lambda_{ \pm ; p-1} \Lambda_{ \pm ; p} .
$$

Let us recall now that the Recursion Operators arise. Suppose we are looking for the NLEEs that have Lax representation $[L, A]=0$ with $L$ being the CBC system operator and $A$ is the form (5). Then from the condition $[L, A]=0$ we first obtain $A_{n-1}=\operatorname{ad}_{J}^{-1}[q, A]$ and next for $0<k<n-1$ the recursion relations $(6,7)$ from which follow the NLEES (8) related to $L$. Let us assume now that we have a $\mathbb{Z}_{p}$ reduction of the type we discussed. Then we have $q \in \overline{\mathfrak{g}}^{[0]}, J \in \mathfrak{h}^{[1]}$. Next from (17) we see that we must have $\mathcal{K}\left(A_{s}\right)=\omega^{s} A_{s}$. Assume that $A_{n} \in \mathfrak{h}^{[n]}$. Then $A_{n-1} \in \overline{\mathfrak{g}}^{[n-1]}$ and from the equations (6), (7) we see that $A_{s} \in \mathfrak{g}^{[s]}$. Therefore the reduction requirements will be satisfied automatically when we choose $A_{n} \in \mathfrak{h}^{[n]}$. Since $n$ is a natural number let us write it into the form $n=k p+m$ where $k, p, m$ are natural numbers and $0 \leq m<p$. Then

$$
\begin{aligned}
\Lambda_{ \pm}^{n} \operatorname{ad}_{J}^{-1}\left[A_{n}, q\right]=\Lambda_{ \pm}^{k p} & \Lambda_{ \pm}^{m} \operatorname{ad}_{J}^{-1}\left[A_{n}, q\right] \\
& =\left(\Lambda_{ \pm ; 0} \ldots \Lambda_{ \pm ; p-1}\right)^{k} \Lambda_{ \pm ; 0} \ldots \Lambda_{ \pm ; m-1} \operatorname{ad}_{J}^{-1}\left[A_{n}, q\right]
\end{aligned}
$$

Starting from the work Fordy and Gibbons $[5,6]$ it is frequently said that when reductions are present the Recursion Operator becomes of higher order in the derivative and factorizes into first order differential operators. The formula (32) also is used in order to claim that the Recursion Operators $R_{ \pm}$in the presence of $\mathbb{Z}_{p}$ reduction factors

$$
R_{ \pm}=\Lambda_{ \pm ; 0} \ldots \Lambda_{ \pm ; p-2} \Lambda_{ \pm ; p-1} .
$$

This of course is true but in fact in our case the Recursion Operator is a power of the Recursion Operator for the CBC system in general position and the "factors" $\Lambda_{ \pm ; s}$ are restrictions of the Recursion Operator in general position on some subspaces

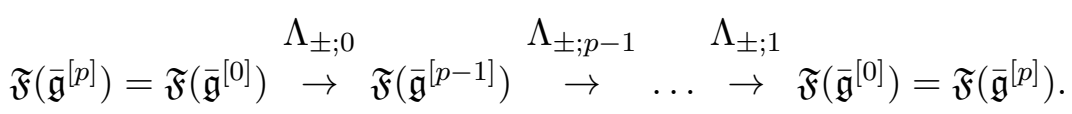


Though simple, the above remarks about the "factorization" will be crucial for the geometric picture.

Remark 3. Of course, if one writes explicitly the projections onto the spaces $\mathfrak{g}^{[s]}$ it might be a little difficult to recognize that $\Lambda_{ \pm ; s}$ are just the restrictions of $\Lambda_{ \pm}$, see [9], where these operators have been calculated in the case of the simple algebras $B_{n}$ and $C_{n}$.

\section{Geometry of the Recursion Operators}

\subsection{Poisson-Nijenhuis Structures Related to CBC Sysem}

Let us remind first the essentials of the geometric picture related to the Recursion Operators in general position. We just outline here the theory assuming that all the important notions such as Poisson structure and Poisson-Nijenhuis structure on a manifold (P-N structure) are known. The missing details and proofs can be found in [8] from where we reproduce some of the facts for the Recursion Operators for CBC system in general position.

The P-N manifolds arise usually if one has compatible Poisson tensor fields $P, Q$ on a manifold $\mathcal{M}$. Of course for $q \in \mathcal{M}$

$$
P_{q}, Q_{q}: T_{q}^{*}(\mathcal{M}) \mapsto T_{q}(\mathcal{M})
$$

where by $T_{q}(\mathcal{M})$ and $T_{q}^{*}(\mathcal{M})$ are denoted the tangent and the cotangent spaces at $q$. The fact that $P, Q$ are compatible means that $P+Q$ is also a Poisson tensor. In fact it is known that in this case we have even a family of Poisson tensors $a P+b Q$ ( $a, b$ are constants). Then in case $Q$ is invertible ( $q \mapsto Q_{q}^{-1}$ exists and is smooth) we can define a tensor field $N=P Q^{-1}$

$$
N_{q}: T_{q}(\mathcal{M}) \mapsto T_{q}(\mathcal{M}) .
$$

Then the pair $(Q, N)$ endows $\mathcal{M}$ with a P-N structure. This result is due to $\mathrm{F}$. Magri who was the first to understand the relevance of the P-N structure to the soliton equations theory, see $[17,18]$ and $[8]$ where there is extensive bibliography. Finally, if we have some manifold $\mathcal{M}$ we shall denote by the same symbol the two-forms $\omega_{q}$ at $q \in \mathcal{M}$ and the corresponding fields $\bar{\omega}_{q}: T_{q}(\mathcal{M}) \mapsto T_{q}^{*}(\mathcal{M})$ of linear maps such that

$$
\omega_{q}(\xi, \eta)=\left\langle\bar{\omega}_{q}(\xi), \eta\right\rangle_{0}, \quad \xi, \eta \in T_{q}(\mathcal{M}) .
$$

Here by $\langle,\rangle_{0}$ is denoted the canonical pairing between a linear space and its dual. Thus two-forms and Poisson tensor fields will appear as fields of operators. 
Since very frequently the Poisson tensors are not invertible for performing the above construction it becomes important to restrict them on submanifolds where they are invertible. The question how to restrict a Poisson structure on a submanifold has been given complete solution in [21], see also [25,26]. We shall use a simplified version of the results obtained in these papers which has been introduced in $[19,20]$ and we shall call it First Restriction Theorem

Theorem 4. Let $\mathcal{M}$ be Poisson manifold with Poisson tensor $P$ and $\overline{\mathcal{M}} \subset \mathcal{M}$ be a submanifold. Let us denote by $\mathrm{j}$ the inclusion map of $\overline{\mathcal{M}}$ into $\mathcal{M}$, by $\mathcal{X}_{P}^{*}(\overline{\mathcal{M}})_{m}$ the subspace of covectors $\alpha \in T_{m}^{*}(\mathcal{M})$ such that

$$
P_{m}(\alpha) \in \operatorname{dj}_{\mathrm{m}}\left(T_{m}(\overline{\mathcal{M}})\right)=\operatorname{im}\left(\mathrm{dj}_{\mathrm{m}}\right), \quad m \in \overline{\mathcal{M}}
$$

where im denotes the image and by $T^{\perp}(\overline{\mathcal{M}})_{m}$ - the set of all covectors at $m \in$ $\mathcal{M}$ vanishing on the subspace $\operatorname{im}\left(\mathrm{dj}_{\mathrm{m}}\right), m \in \overline{\mathcal{M}}$ (also called the annihilator of $\operatorname{im}\left(\mathrm{dj}_{\mathrm{m}}\right)$ in $\left.T_{m}^{*}(\mathcal{M})\right)$. Let the following relations hold

$$
\begin{array}{ll}
\mathcal{X}_{P}^{*}(\overline{\mathcal{M}})_{m}+T^{\perp}(\overline{\mathcal{M}})_{m}=T_{m}^{*}(\mathcal{M}), & m \in \overline{\mathcal{M}} \\
\mathcal{X}_{P}^{*}(\overline{\mathcal{M}})_{m} \cap T^{\perp}(\overline{\mathcal{M}})_{m} \subset \operatorname{ker}\left(P_{m}\right), & m \in \overline{\mathcal{M}} .
\end{array}
$$

Then there exists unique Poisson tensor $\bar{P}$ on $\overline{\mathcal{M}}$, j-related with $P$, that is

$$
P_{m}=\mathrm{dj}_{\mathrm{m}} \circ \bar{P}_{m} \circ\left(\mathrm{dj}_{\mathrm{m}}\right)^{*}, \quad m \in \overline{\mathcal{M}} .
$$

The proof of the theorem is constructive, one takes $\beta \in T_{m}^{*}(\overline{\mathcal{M}})$, then represents $\left(\mathrm{j}^{*} \beta\right)_{\mathrm{m}}$ as $\alpha_{1}+\alpha_{2}$ where $\alpha_{1} \in \mathcal{X}_{P}^{*}(\overline{\mathcal{M}})_{m}, \alpha_{2} \in T^{\perp}(\overline{\mathcal{M}})_{m}$ and puts $\bar{P}_{m}(\beta)=$ $P_{m}\left(\alpha_{1}\right)$ (we identify $m$ and $\mathrm{j}(\mathrm{m})$ here).

Let $\mathfrak{g}$ be the semisimple Lie algebra on which the CBC system is defined. For a subspace $\mathfrak{a} \subset \mathfrak{g}$ let us denote by $\mathfrak{F}(\mathfrak{a})$ the set of smooth, fast decreasing functions $f: \mathbb{R} \rightarrow \mathfrak{g}$. We have for example the set of functions $\mathfrak{F}(\mathfrak{h})$ with values in the Cartan subalgebra $\mathfrak{h} \subset \mathfrak{g}$ and the set of functions $\mathfrak{F}(\overline{\mathfrak{g}})$ of the functions with values in the orthogonal complement of $\mathfrak{h}$. Clearly, $\mathfrak{F}(\mathfrak{g})$ and $\mathfrak{F}(\mathfrak{h})$ are Lie algebras too if we define the Lie bracket of two functions $f, g$ point-wise, that is we put

$$
[f, g](x)=[f(x), g(x)], \quad x \in \mathbb{R} .
$$

Admitting some lack of rigor we shall identify $\mathfrak{F}(\mathfrak{g})$ with $\mathfrak{F}(\mathfrak{g})^{*}$ using the bilinear form

$$
\langle\langle X, Y\rangle\rangle=\int_{-\infty}^{+\infty}\langle X(x), Y(x)\rangle \mathrm{d} x, \quad X, Y \in \mathfrak{F}(\mathfrak{g})
$$

where $\langle\xi, \eta\rangle=\operatorname{tr}\left(\operatorname{ad}_{\xi} \circ \operatorname{ad}_{\eta}\right), \xi, \eta \in \mathfrak{g}$ is the Killing form of the algebra $\mathfrak{g}$. 
Now, taking into account the conventions and identifications we made, we note that as it is well known for the equations that can be solved with the help of auxiliary linear problem (2) we have the following compatible Poisson tensors (one can obtain this fact as a consequence from some general algebraic construction, see for example $[3,8]$

$$
\begin{aligned}
& Q_{q}^{0}(\xi)=-\operatorname{ad}_{\xi} J, \quad q(x), \xi(x) \in \mathfrak{F}(\mathfrak{g}) \\
& P_{q}^{0}(\xi)=-\operatorname{ad}_{\xi} q+\mathrm{i} \partial_{x} \xi, \quad q(x), \xi(x) \in \mathfrak{F}(\mathfrak{g}) .
\end{aligned}
$$

To see that these tensors, which are defined on the manifold $\mathcal{M}=\mathfrak{F}(\mathfrak{g})^{*}$, are tensors of the type we want, we note that since $\mathcal{M}$ is linear space the tangent space at each point coincides with $\mathfrak{F}(\mathfrak{g})^{*}$ and the cotangent space with $\mathfrak{F}(\mathfrak{g})^{* *}$. Due to the convention we have made to identify vectors and covectors through (39), we can assume that both these spaces coincide with $\mathfrak{F}(\mathfrak{g})$. Then in $(40), q \in \mathfrak{F}(\mathfrak{g})^{*}$, $\xi \in T_{q}(\mathcal{M}) \sim \mathfrak{F}(\mathfrak{g})$. It is easy to notice that the tensor $Q^{0}$ is not kernel free and therefore we cannot find $\left(Q^{0}\right)^{-1}$. Fortunately one can restrict $Q$ on some integral leaf of the distribution $\operatorname{im}\left(Q^{0}\right)$ and then the restricted tensor will be nondegenerate. So we applying the construction of the Restriction Theorem (4) to the tensor $Q^{0}$. Considering the distribution

$$
q \rightarrow \operatorname{im}\left(Q^{0}\right)_{q}=\operatorname{im}\left(\operatorname{ad}_{J}\right)
$$

(where im denotes the image) we see that he elements of $\mathrm{im}\left(\operatorname{ad}_{J}\right)$ are the functions belonging to $\mathfrak{F}(\mathfrak{g})$ taking values in the orthogonal complement $\overline{\mathfrak{g}}$ of the Cartan subalgebra $\mathfrak{h} \subset \mathfrak{g}$ with respect to the Killing form (recall that $J$ is regular). We shall denote the subspace of this elements by $\mathfrak{F}(\overline{\mathfrak{g}})$. Then the integral leaves of the distribution (41) are the submanifolds

$$
\overline{\mathcal{M}}_{c}=\{q ; q=c+\xi, c-\text { fixed, } c, \xi \in \mathfrak{F}(\overline{\mathfrak{g}})\} .
$$

Let us choose the leaf

$$
\left.\overline{\mathcal{M}}_{c}\right|_{c=0}=\overline{\mathcal{M}}_{0}=\mathfrak{F}(\overline{\mathfrak{g}})
$$

and let $\mathrm{j}: \overline{\mathcal{M}}_{0} \mapsto \mathfrak{F}(\mathfrak{g})$ be the inclusion map. Clearly $T_{q}\left(\overline{\mathcal{M}}_{0}\right)=\mathfrak{F}(\overline{\mathfrak{g}})$ and having in mind the pairing (39) we can also assume that $T_{q}^{*}\left(\overline{\mathcal{M}}_{0}\right)=\mathfrak{F}(\overline{\mathfrak{g}})$. If $\alpha \in T_{q}^{*}(\mathcal{M})$ then $\pi_{0} \alpha=\alpha$, where $\pi_{0}$ is the orthogonal projector (with respect to the Killing form) onto the space $\overline{\mathfrak{g}}$. Due to the identifications we have made $\mathrm{dj}=\pi_{0}$ and $[\mathrm{dj}]^{*}=\pi_{0}$. All this means that we can write $[\mathrm{dj}]^{*} \alpha=\alpha$ and then the Restriction Theorem means that

$$
Q(\alpha)=Q^{0}\left([\mathrm{dj}]^{*} \alpha\right)=\mathrm{Q}^{0}(\alpha) .
$$

Thus $Q=\operatorname{ad}_{J}$. Note that now the tensor $Q$ is nondegenerate, since on the space $\overline{\mathfrak{g}}$ there exists the inverse of $\operatorname{ad}_{J}$ - the operator $\operatorname{ad}_{J}^{-1}$. 
To restrict $P^{0}$ we again invoke the Restriction Theorem (4). We have seen that in order to perform the restriction on the submanifold $\overline{\mathcal{M}}_{0}$ the following conditions must hold

$$
\begin{array}{ll}
\chi_{P^{0}}^{*}\left(\overline{\mathcal{M}}_{0}\right)_{q}+T^{\perp}\left(\overline{\mathcal{M}}_{0}\right)_{q}=T_{q}^{*}(\mathcal{M}), & q \in \overline{\mathcal{M}}_{0} \\
\chi_{P^{0}}^{*}\left(\overline{\mathcal{M}}_{0}\right)_{q} \cap T^{\perp}\left(\overline{\mathcal{M}}_{0}\right)_{q} \subset \operatorname{ker}\left(P_{q}\right), & q \in \overline{\mathcal{M}}_{0} .
\end{array}
$$

A simple calculation shows that

$$
T^{\perp}\left(\overline{\mathcal{M}}_{0}\right)_{q}=\left\{\alpha ; \alpha \in T_{q}^{*}(\mathcal{M}),\langle\langle\alpha, \xi\rangle\rangle=0, \xi \in \mathfrak{F}(\overline{\mathfrak{g}})\right\} .
$$

In other words, $T^{\perp}\left(\overline{\mathcal{M}}_{0}\right)_{q}$ consists of functions taking values in $\mathfrak{h}$ and it is natural to denote the space of these functions by $\mathfrak{F}(\mathfrak{h})$.

From the other hand

$$
\chi_{P^{0}}^{*}\left(\overline{\mathcal{M}}_{0}\right)_{q}=\left\{\alpha ; \alpha \in T_{q}^{*}(\mathcal{M}), \mathrm{i} \partial_{x} \alpha+[q, \alpha] \in \mathfrak{F}(\overline{\mathfrak{g}})\right\} .
$$

Therefore $\alpha \in \chi_{P^{0}}^{*}\left(\overline{\mathcal{M}}_{0}\right)_{q}$ exactly when

$$
\left(\mathbf{1}-\pi_{0}\right)\left(\mathrm{i} \partial_{x} \alpha+[q, \alpha]\right)=0 .
$$

If $\alpha \in \chi_{P^{0}}^{*}\left(\overline{\mathcal{M}}_{0}\right)_{q} \cap T^{\perp}\left(\overline{\mathcal{M}}_{0}\right)_{q}$, then $[\mathfrak{h}, \overline{\mathfrak{g}}] \subset \overline{\mathfrak{g}}$ shows that $\partial_{x} \alpha=0$. Since we have $\lim _{x \rightarrow \pm \infty} \alpha(x)=0$ we get $\alpha=0$. Thus we have proved that

$$
T^{\perp}\left(\overline{\mathcal{M}}_{0}\right)_{q} \cap \chi_{P^{0}}^{*}\left(\overline{\mathcal{M}}_{0}\right)_{q}=\{0\}
$$

and the requirement (46) of the Restriction Theorem is fulfilled.

In order to prove that (45) is also true, let us remark that the condition (49) can be cast into the form

$$
\left(\mathbf{1}-\pi_{0}\right) \alpha=\mathrm{i}\left(\mathbf{1}-\pi_{0}\right) \int_{-\infty}^{x}[q(y), \alpha(y)] \mathrm{d} y+A(\alpha, q)
$$

where $A(\alpha, q)$ is some constant in $x$ which in general can depend on $\alpha$ and the potential $q$. Since $\lim _{x \rightarrow \pm \infty} \alpha(x)=0$, we must have

$$
A(q, \alpha)=\mathrm{i}\left(\mathbf{1}-\pi_{0}\right) \int_{-\infty}^{+\infty}[q(y), \alpha(y)] \mathrm{d} y=0
$$

or equivalently

$$
\langle\langle[H, q], \alpha\rangle\rangle=0, \quad H \in \mathfrak{h} .
$$


The above relations impose implicit restrictions on the cotangent vectors on $\overline{\mathcal{M}}_{0}$. Actually, if we choose a basis $\left\{H_{i}\right\}_{1}^{r}$ in $\mathfrak{h}$ then (53) is equivalent to $r$ equations $\left\langle\left\langle\left[H_{i}, q\right], \alpha\right\rangle\right\rangle=0, i=1,2 \ldots, r$. If we want to continue with the geometric constructions we are forced to assume that the (53) is fulfilled. In order to stress that for our potentials we need to have the relations (53) in what follows we shall denote the manifold of potentials by $\mathcal{M}_{0}$.

One can prove that $\mathcal{M}_{0}$ is dense in $\overline{\mathcal{M}}_{0}$, but we shall not go into this matter. We simply want to prove that (45), (46) are true for the manifold $\mathcal{M}_{0}$ instead of $\overline{\mathcal{M}}_{0}$.

Let us consider now the requirement (45). For arbitrary $\alpha \in T_{q}^{*}\left(\mathcal{M}_{0}\right)$ we put

$$
\gamma(\alpha)=-\mathrm{i}\left(\mathbf{1}-\pi_{0}\right) \int_{-\infty}^{x}\left[q(y), \pi_{0}(\alpha)(y)\right] \mathrm{d} y .
$$

It is not difficult to notice that

$$
\pi_{0}(\alpha)-\gamma(\alpha) \in \chi_{P^{0}}^{*}\left(\mathcal{M}_{0}\right)_{q}
$$

For that reason the identity

$$
\alpha=\left(\pi_{0}(\alpha)-\gamma(\alpha)\right)+\left(\left(\mathbf{1}-\pi_{0}\right) \alpha+\gamma(\alpha)\right)
$$

shows that

$$
\chi_{P^{0}}^{*}\left(\mathcal{M}_{0}\right)_{q} \oplus T^{\perp}\left(\mathcal{M}_{0}\right)_{q}=T_{q}^{*}(\mathcal{M}) .
$$

Thus the conditions of the Restriction Theorem hold and $P^{0}$ allows restriction on $\mathcal{M}_{0}$. Let us denote this restriction by $P$. Now we are going to calculate it. Let $\alpha \in T_{q}^{*}\left(\mathcal{M}_{0}\right)$. As before $[\mathrm{dj}]^{*} \alpha=\alpha$ and according to the restriction procedure we must put

$$
P(\alpha)=P^{0}(\alpha-\gamma(\alpha)) .
$$

It is easy to calculate that

$$
\begin{aligned}
& P(\alpha)=\mathrm{i} \partial_{x} \alpha+\pi_{0}([q, \alpha])+\left[q, \mathrm{i}\left(\mathbf{1}-\pi_{0}\right) \int_{-\infty}^{x}[q, \alpha](y) \mathrm{d} y\right] \\
& \pi_{0}(\alpha)=\alpha \in T_{q}^{*}\left(\mathcal{M}_{0}\right) .
\end{aligned}
$$

Now it is possible to obtain the Nijenhuis tensor $N=P \circ Q^{-1}=P Q^{-1}$

$$
N=\left[\mathrm{i} \partial_{x}+\pi_{0} \operatorname{ad}_{q}+\operatorname{iad}_{q}\left(\mathbf{1}-\pi_{0}\right) \int_{-\infty}^{x} \operatorname{ad}_{q} \cdot \mathrm{d} y\right]\left(\operatorname{ad}_{J}\right)^{-1} .
$$


We would like also to note that as a consequence from the condition (53) in the lower bound of the above integral we could write $+\infty$ instead of $-\infty$ without changing the value of the expressions for $P$ and $N$, for this reason frequently instead of the integral is written $\partial_{x}^{-1}$.

We also can put the above in an equivalent form

$$
N=\left[\mathrm{i} \partial_{x}+\pi_{0} \operatorname{ad}_{q}+\operatorname{iad}_{q}\left(\mathbf{1}-\pi_{0}\right) \partial_{x}^{-1} \operatorname{ad}_{q}\right] \operatorname{ad}_{J}^{-1} .
$$

Now the tensor fields $P$ and $N$ endow $\mathcal{M}_{0}$ with a P-N structure. The adjoint of $\mathrm{N}$ is easily found

$$
N^{*}=\left(P Q^{-1}\right)^{*}=\left(Q^{*}\right)^{-1} P^{*}=Q^{-1} P=Q^{-1}\left(P Q^{-1}\right) Q=Q^{-1} N Q .
$$

Now, after all this geometric theory, the comparison shows that $N^{*}$ is exactly equal to the generating operators $\Lambda_{ \pm}$for the CBC system. We can also write

$$
N^{*}=\frac{1}{2}\left(\Lambda_{+}+\Lambda_{-}\right)=\Lambda
$$

The geometric theory however is incomplete without the possibility to calculate the fundamental fields of the P-N structure. One can show, see [8], that the vector fields

$$
X_{H}: q \rightarrow X_{H}(q)=[H, q], \quad H \in \mathfrak{h}
$$

are fundamental fields. The corresponding fundamental forms are

$$
\alpha_{H}: q \rightarrow \alpha_{H}(q)=\operatorname{ad}_{J}^{-1}[H, q] .
$$

(Recall that from the results of part I it follows that the forms $\operatorname{ad}_{J}^{-1}[H, q]$ and $\Lambda_{ \pm} \operatorname{ad}_{J}^{-1}[H, q]$ are closed.) In addition, from the relation

$$
\left[X_{H_{1}}, X_{H_{2}}\right](q)=X_{\left[H_{1}, H_{2}\right]}(q)
$$

it follows that if $H_{1}, H_{2} \in \mathfrak{h}$ then the Lie bracket of the fields $X_{H_{1}}$ and $X_{H_{1}}$ is zero, or equivalently, that the forms $\alpha_{H_{1}}$ and $\alpha_{H_{2}}$ are in involution. Then the properties of the P-N structure easily lead to

Proposition 5. Let all quantities be as defined in the above. Then

- The vector fields $N^{n} X_{H}, n=0,1, \ldots, H \in \mathfrak{h}$ commute.

- The equations

$$
\operatorname{iad}_{J}^{-1} q_{t}+\Lambda^{n} \operatorname{ad}_{J}^{-1}[H, q]=0
$$

are Hamiltonian with respect to the hierarchy of symplectic structures: $\Omega^{n}=$ $\Lambda^{n}\left(Q^{0}\right)^{-1}=\Lambda^{n} \operatorname{ad}_{J}^{-1}$.

This proposition gives geometric interpretation of the Recursion Operators for the $\mathrm{CBC}$ system in general position. 


\subsection{Poisson-Nijenhuis Structures for CBC System with Reductions}

When there is a $\mathbb{Z}_{p}$ reduction defined by the automorphism $\mathcal{K}$ of finite order $p$ the manifold of potentials is restricted as described in the above. The eigenvalues of $\mathcal{K}$ are $\omega^{s}, s=0,1, \ldots, p-1, \omega=\exp \frac{2 \pi \mathrm{i}}{p}$ and to them correspond the eigenspaces $\mathfrak{g}^{[s]}$. Since for $\mathcal{K}$ the spaces $\mathfrak{h}$ and $\overline{\mathfrak{g}}$ are invariant the eigenspace $\mathfrak{g}^{[s]}$ splits into $\overline{\mathfrak{g}}^{[s]} \oplus \mathfrak{h}^{[s]}, \overline{\mathfrak{g}}^{[s]} \subset \overline{\mathfrak{g}}, \mathfrak{h}^{[s]} \subset \mathfrak{h}$ so that $\overline{\mathfrak{g}}=\oplus_{s=0}^{p-1} \overline{\mathfrak{g}}^{[s]}, \mathfrak{h}=\oplus_{s=0}^{p-1} \mathfrak{h}^{[s]}$

We have that $J \in \mathfrak{h}^{[1]}$ and $q \in \mathfrak{F}\left(\overline{\mathfrak{g}}^{[0]}\right)$ so

$$
\operatorname{ad}_{J}\left(\overline{\mathfrak{g}}^{[s]}\right) \subset \overline{\mathfrak{g}}^{[s+1]}, \quad \operatorname{ad}_{J}^{-1}\left(\overline{\mathfrak{g}}^{[s]}\right) \subset \overline{\mathfrak{g}}^{[s-1]}, \quad \operatorname{ad}_{q}\left(\mathfrak{g}^{[s]}\right) \subset \mathfrak{g}^{[s]} .
$$

The superscripts are understood modulo $p$. It also means that now the manifold of potentials is restricted from $\mathfrak{F}(\overline{\mathfrak{g}})$ to $\mathcal{N}=\mathfrak{F}\left(\overline{\mathfrak{g}}^{[0]}\right)$. Since $\mathcal{N}$ is a vector space we identify its tangent spaces at some $q$ with $\mathfrak{F}\left(\overline{\mathfrak{g}}^{[0]}\right)$. As before, using the nondegeneracy of the form $\langle\langle\rangle$,$\rangle we identify with \mathfrak{F}\left(\overline{\mathfrak{g}}^{[0]}\right)$ also the cotangent spaces.

Looking at the expression (59) we see that for $X \in \mathfrak{F}\left(\overline{\mathfrak{g}}^{[s]}\right)$

$$
N X=\left[\operatorname{i} \partial_{x} X+\pi_{0} \operatorname{ad}_{q}+\operatorname{iad}_{q}\left(\mathbf{1}-\pi_{0}\right) \partial_{x}^{-1} \operatorname{ad}_{q}\right] \operatorname{ad}_{J}^{-1} X \in \mathfrak{F}\left(\overline{\mathfrak{g}}^{[s-1]}\right)
$$

so that

$$
N \mathfrak{F}\left(\overline{\mathfrak{g}}^{[s]}\right) \subset \mathfrak{F}\left(\overline{\mathfrak{g}}^{[s-1]}\right), \quad \operatorname{ad}_{J} \mathfrak{F}\left(\overline{\mathfrak{g}}^{[s]}\right)=\mathfrak{F}\left(\overline{\mathfrak{g}}^{[s-1]}\right) .
$$

In particular,

$$
N\left(T_{q}(\mathcal{N})\right)=N \mathfrak{F}\left(\overline{\mathfrak{g}}^{[0]}\right) \subset \mathfrak{F}\left(\overline{\mathfrak{g}}^{[p-1]}\right) .
$$

It follows that $N$ does not allow restriction on $\mathcal{N}$. However, as easily seen $N^{p}$ allows such a restriction and is of course a Nijenhuis tensor on $\mathcal{N}$. Similarly, one sees that the Poisson tensor field $Q=\operatorname{ad}_{J}$ restricted on $\mathcal{N}$ reduces to zero. Of course then one can try to restrict $P$. Looking at it, we see that $\alpha \in \mathfrak{F}(\overline{\mathfrak{g}})=$ $T_{q}^{*}\left(\mathcal{M}_{0}\right)$ will belong to $\mathcal{X}_{P}^{*}(\mathcal{N})_{q}$ (the notation we use here is that of the First Restriction Theorem) if

$$
P(\alpha)=\mathrm{i} \partial_{x} \alpha+\pi_{0}([q, \alpha])+\left[q, \mathrm{i}\left(\mathbf{1}-\pi_{0}\right) \partial_{x}^{-1}[q, \alpha](y)\right] \in T_{q}^{*}(\mathcal{N})=\mathfrak{F} \cdot\left(\overline{\mathfrak{g}}^{[0]}\right)
$$

Next,

$$
T^{\perp}(\mathcal{N})_{q}=\left\{\alpha ; \alpha \in \mathfrak{F}(\overline{\mathfrak{g}}),\langle\langle\alpha, \xi\rangle\rangle=0, \xi \in \mathfrak{F}\left(\overline{\mathfrak{g}}^{[0]}\right)\right\} .
$$

It is not hard to establish that $\mathcal{X}_{P}^{*}(\mathcal{N})_{q}=\mathfrak{F}\left(\overline{\mathfrak{g}}^{[0]}\right)$ and $T^{\perp}(\mathcal{N})_{q}=\mathfrak{F}\left(\oplus_{s=1}^{p-1} \overline{\mathfrak{g}}^{[s]}\right)$ so one sees that

$$
\mathcal{X}_{P}^{*}(\mathcal{N})_{q}+T^{\perp}(\mathcal{N})_{q}=T_{q}\left(\mathcal{M}_{0}\right), \quad \mathcal{X}_{P}^{*}(\mathcal{N})_{q} \cap T^{\perp}(\mathcal{N})_{q}=\{0\} .
$$

Thus applying the First Restriction Theorem we we get that $P$ can be restricted and the restriction $\bar{P}$ has the same form. 
Since $P$ allows a restriction on $\mathcal{N}$ one can apply the following simple results from the general theory of the P-N manifolds and from the restriction theory of these manifolds, see $[8,19,20]$

Theorem 6. If $\mathcal{M}$ is a $\mathrm{P}-\mathrm{N}$ manifold endowed with Poisson structure $\mathrm{P}$ and $\mathrm{Ni}$ jenhuis tensor $N$ then for $k, s=1,2, \ldots$ each pair $\left(N^{k} P=\left(N^{*}\right)^{k} P, N^{s}\right)$ also endows $\mathcal{M}$ with $\mathrm{P}-\mathrm{N}$ structure.

Theorem 7. Let $\mathcal{M}$ be a P-N manifold endowed with Poisson tensor $\mathrm{P}$ and $\mathrm{Ni}$ jenhuis tensor $N$. Let $\overline{\mathcal{M}} \subset \mathcal{M}$ be a submanifold of $\mathcal{M}$ and suppose that we have

i) $P$ allows restriction $\bar{P}$ on $\overline{\mathcal{M}}$, that is $\bar{P}$ is a Poisson tensor such that if $\mathrm{j}: \overline{\mathcal{M}} \mapsto \mathcal{M}$ is the inclusion map then $\bar{P}$ is j-related with $P$

$$
P_{m}=\mathrm{dj}_{m} \circ \bar{P}_{m} \circ\left(\mathrm{dj}_{m}\right)^{*}, \quad m \in \overline{\mathcal{M}} .
$$

ii) The tangent spaces of $\overline{\mathcal{M}}$, considered as subspaces of the tangent spaces of $\mathcal{M}$ are invariant under $N$, so that $N$ allows a natural restriction $\bar{N}$ to $\overline{\mathcal{M}}$, that is $\bar{N}$ is j-related with $N$.

Then $(\bar{P}, \bar{N})$ endow $\overline{\mathcal{M}}$ with a P-N structure.

We call the above theorem Second Restriction Theorem.

As a result, we have that on $\mathcal{N}=\mathfrak{F}\left(\overline{\mathfrak{g}}^{[0]}\right)$ the tensors $P$ given by $(57)$ and $N^{p}$, where $N$ is given by (59), allow restriction. They do not change their form and endow $\mathcal{N}$ with P-N structure. It is also immediate to see that $\left(N^{p}\right)^{*}=\Lambda_{ \pm}^{p}$.

The last thing that remains to be done is to calculate the integrable equations (fundamental fields) related with the P-N structure we just introduced. Clearly not all the fields from the hierarchies generated by the fields $X_{H}=[H, q], H \in \mathfrak{h}$ (see (61)) will be tangent to the submanifold $\mathcal{N}$ so we must find which of the fields from the hierarchies 'survive' the reduction. It is obvious that in order that $X_{H}$ be tangent to $\mathcal{N}$ one needs $H \in \mathfrak{h}^{[0]}$. Then if we assume that from the hierarchy $N^{k} X_{H}, k=0,1,2, \ldots$ 'survive' only the members $N^{p n} X_{H}, n=0,1,2, \ldots$ and these fields because they are fundamental for the tensors $P$ and $N$ are fundamental for the P-N structure on $\mathcal{N}$. But these are not the only fields from the hierarchy in general position that should be taken. As a matter of fact one easily sees that if $H \in \mathfrak{h}^{[s]}, s=0,1, \ldots p-1$ then $N^{s} X_{H} \in \mathfrak{F}\left(\overline{\mathfrak{g}}^{[0]}\right)$, that is, this field is tangent to $\mathcal{N}$. We arrive therefore to the result that the hierarchies of fundamental fields are

$$
N^{k p+s} X_{H}, \quad H \in \mathfrak{h}^{[s]}, \quad s=0,1, \ldots p-1, \quad k=0,1,2, \ldots
$$


(In fact the general hierarchies are obtained taking finite linear combinations of these fields with constant coefficients but this is the usual way the things are described.) Taking into account that the new Nijenhuis tensor is now $N^{p}$ we see that its action on the fields

$$
N^{s} X_{H}, \quad H \in \mathfrak{h}^{[s]}
$$

generates the fundamental fields of the P-N structure on $\mathcal{N}$. Remembering that $N=\operatorname{ad}_{J} \circ \Lambda \circ \operatorname{ad}_{J}^{-1}$ we arrive at the hierarchy of equations

$$
\operatorname{iad}_{J}^{-1} q_{t}=\Lambda^{k p} \Lambda^{s} \operatorname{ad}_{J}^{-1}[H, q], H \in \mathfrak{h}^{[s]} .
$$

The coefficient " $i$ " is put here in order to have consistency with the formulae obtained through the Lax representation. Thus we have

Theorem 8. For the $\mathrm{CBC}$ system subject to $\mathbb{Z}_{p}$ reduction the Recursion Operator is equal to $\Lambda_{ \pm}^{p}$ where $\Lambda_{ \pm}$is the recursion operator for the CBC system in general position. The operator $\Lambda_{ \pm}^{p}$ allows the usual geometric interpretation - it is the dual of Nijenhuis operator for some P-N structure and the corresponding NLEEs have the usual interpretation - they are the fundamental fields of this structure.

\section{Conclusion}

We have seen that the observed factorization of the Recursion Operators for the CBC system in the presence of $\mathbb{Z}_{p}$ reductions in fact means that the role of the Recursion Operator is played now by the $p$-th power of the Recursion Operator for the $\mathrm{CBC}$ system in general position. This allows clear and simple geometric interpretation in terms of Poisson-Nijenhuis structures since each power of a Nijenhuis tensor is also a Nijenhuis tensor.

\section{References}

[1] Ablowitz M., Kaup D., Newell A. and Segur H., The Inverse Scattering Transform - Fourier Analysis for Nonlinear Problems, Studies in Appl. Math. 53 (1974) 249-315.

[2] Beals R. and Coifman R., Scattering and Inverse Scattering for First Order Systems, Comm. Pure \& Appl. Math. 37 (1984) 39-89.

[3] Drinfeld V. and Sokolov V., Lie Algebras and Korteweg-de Vries Type Equations, VINITI Series: Contemporary Problems of Mathematics. Recent Developments, Moscow 1985. 
[4] Faddeev L. and Takhtadjan L., Hamiltonian Method in the Theory of Solitons, Springer, Berlin 1987.

[5] Fordy A. and Gibbons J., Factorization of Operators I. Miura Transformations, J. Math. Phys. 21 (1980) 2508-2510.

[6] Fordy A. and Gibbons J., Factorization of Operators II, J. Math. Phys. 22 (1981) 1170-1175.

[7] Gerdjikov V. and Yanovski A., Completeness of the Eigenfunctions for the Caudrey-Beals-Coifman System, J. Math. Phys. 35 (1994) 3687-3721.

[8] Gerdjikov V., Vilasi G. and Yanovski A., Integrable Hamiltonian Hierarchies - Spectral and Geometric Methods, Springer, Heidelberg 2008.

[9] Gerdjikov V., Kostov N. and Valchev T., Generalized Zakharov-Shabat Systems and Nonlinear Evolution Equations with Deep Reductions, In: BGSIAM'09, S. Margenov, S. Dimova and A. Slavova (Eds), Demetra, Sofia 2010, pp 51-57.

[10] Gerdjikov V., Mikhailov A. and Valchev T., Reductions of Integrable Equations on A.III-Symmetric Spaces, J. Phys. A: Math. \& Theor. 43 (2010) 434015 .

[11] Gerdjikov V., Mikhailov A. and Valchev T., Recursion Operators and Reductions of Integrable Equations on Symmetric Spaces, J. Geom. Symmetry Phys. 20 (2010) 1-34.

[12] Gerdjikov V., Grahovski G., Mikhailov A. and Valchev T., Polynomial Bundles and Generalized Fourier Transforms for Integrable Equations on A. IIItype Symmetric Spaces, SIGMA 7 (2011) 096.

[13] Goto M. and Grosshans F., Semisimple Lie Algebras, Lecture Notes in Pure and Applied Mathematics vol. 38, M. Dekker, New York 1978.

[14] Grahovski G., On the Reductions and Scattering Data for the CBC System, In: Geometry, Integrability and Quantization III, I. Mladenov and G. Naber (Eds), Coral Press, Sofia 2002, pp 262-277.

[15] Grahovski G., On the Reductions and Scattering Data for the Generalized Zakharov-Shabat Systems, In: Nonlinear Physics: Theory and Experiment II, M. Ablowitz, M. Boiti, F. Pempinelli and B. Prinari (Eds), World Scientific, Singapore 2003, pp 71-78. 
[16] Iliev I., Khristov E. and Kirchev K., Spectral Methods in Soliton Equations, Pitman Monographs and Surveys in Pure and Applied Mathematics 73, John Wiley, New York 1994.

[17] Magri F., A Simple Model of the Integrable Hamiltonian Equations, J. Math. Phys. 19 (1978) 1156-1162.

[18] Magri F., A Geometrical Approach to the Nonlinear Solvable Equations, Lecture Notes in Physics 120, Springer, Berlin 1980.

[19] Magri F. and Morosi C., A Geometrical Characterization of Integrable Hamiltonian Systems through the Theory of Poisson - Nijenhuis Manifolds, Quaderni del Dipartimento di Matematica, Quaderno S 19, Università di Milano 1984.

[20] Magri F., Morosi C. and Ragnisco O., Reduction Techniques for InfiniteDimensional Hamiltonian Systems: Some Ideas and Applications, Comm. Math. Phys. 99 (1985) 115-140.

[21] Marsden J. and Ratiu T., Reduction of Poisson Manifolds, Lett. Math. Phys. 11 (1986) 161-169.

[22] Mikhailov A., Reduction in the Integrable Systems. Reduction Groups(in Russian), Lett. JETF (Letters to Soviet Journal of Experimental and Theoretical Physics) 32 (1979) 187-192.

[23] Mikhailov A., The Reduction Problem and Inverse Scattering Method, Physica D 2 (1981) 73-117.

[24] Mikhailov A. and Lombardo S., Reductions of Integrable Equations. Dihedral Group, J. Phys. A 37 (2004) 7727-7742.

[25] Ortega J. and Ratiu T., Momentum Maps and Hamiltonian Reduction, Progress in Mathematics 222, Birkhäuser, Boston 2004.

[26] Ortega J. and Ratiu T., Singular Reduction of Poisson Manifolds, Lett. Math. Phys. 46 (1998) 59-372.

[27] Valchev T., On Certain Reduction of Integrable Equations on Symmetric Spaces, In: AIP Conference Proceedings 1340, K. Sekigawa, V. Gerdjikov, Y. Matsushita and I. Mladenov (Eds), Melville, New York 2011, pp 154-163.

[28] Yanovski A., Geometric Interpretation of the Recursion Operators for the Generalized Zakharov-Shabat System in Pole Gauge on the Lie Algebra $A_{2}$, J. Geom. Symmetry Phys. 23 (2011) 97-11. 
[29] Yanovski A., On the Recursion Operators for the Gerdjikov, Mikhailov and Valchev System, J. Math. Phys. 52 (2011) 082703.

[30] Zakharov V. and Konopelchenko B., On the Theory of Recursion Operator, Comm. Math. Phys. 94 (1985) 483-510.

Alexandar B. Ianovsky

Department of Mathematics and

Applied Mathematics

University of Cape Town, Cape Town

Rondebosch 7700

SOUTH AFRICA

E-mail address: Alexandar. Ianovsky@uct.ac.za 